\title{
Azole drug resistance in Candida species
}

Candida albicans is found in the mouth and gastrointestinal tract of a significant proportion of the normal human population. This opportunist pathogen can cause deep-seated infection, which may be localised or disseminated, but is more often seen causing mucosal or cutaneous infection. Although $C$. albicans remains the predominant cause of all forms of human candidosis, $C$. tropicalis has emerged as an important pathogen in neutropenic patients and $C$. parapsilosis infection is common in individuals receiving parenteral nutrition. Other Candida species have also been recognised as significant pathogens.

Imidazole and triazole compounds are amongst the most useful drugs for the treatment of human candidosis. They inhibit the C-14 $\alpha$-sterol demethylation step in the formation of ergosterol, leading to accumulation of methylated sterols which disrupt fungal membrane structure. ${ }^{1}$ The earliest members of this large group of drugs, clotrimazole and miconazole, are useful for topical treatment of superficial candidosis and this has remained the case for most of the similar imidazole compounds that have appeared since. However, some more recent compounds, such as ketoconazole and the triazoles fluconazole and itraconazole, are effective after oral administration and can be used to treat both superficial and deep-seated infection.

Although azole antifungal agents have long been in widespread use, there have been few verified reports of resistant strains of $C$. albicans emerging during treatment. The low incidence of clinical resistance has been attributed to the fact that $C$. albicans is a diploid fungus with no haploid sexual stage. ${ }^{2}$ Thus, unless resistance is a dominant character, resistant strains will seldom appear. Until 1986, the few resistant strains of C. albicans that had been isolated had all been from patients who had received long-term oral treatment with ketoconazole for the uncommon condition, chronic mucocutaneous candidosis, which had relapsed in each case despite adequate blood concentrations of the drug. ${ }^{3-5}$ It is not surprising that these initial reports were disputed, given the extent to which the results of MIC determinations with ketoconazole depend on the conditions under which the tests are done. However, extensive testing of these strains of $C$. albicans has confirmed that they are much less susceptible to a range of azole drugs than other strains of this species. ${ }^{5-8}$ At least two mechanisms of resistance are evident. In one, the azole drug fails to cross the cell envelope. In the other, azole-induced blockade of the C-14 sterol demethylation step in the formation of ergosterol is circumvented. ${ }^{8,9}$

Mucosal forms of candidosis are some of the most common and persistent infections in individuals with HIV infection. Many AIDS patients require long-term treatment for suppression of oral or oesophageal candidosis. Oral ketoconazole has been used widely, but treatment failure and relapse have been common. Although this has usually been associated with malabsorption of the drug in AIDS patients, MIC data have sometimes suggested drug resistance as the cause. ${ }^{10}$ The triazole drug fluconazole is less toxic and better tolerated than ketoconazole, and is absorbed more reliably. It has been shown to be more effective than ketoconazole in controlling oropharyngeal candidosis in AIDS patients. ${ }^{11}$ However, one consequence of the long-term use of fluconazole has been a marked increase in the number of reports of AIDS patients with persistent oral or oesophageal candidosis, apparently because of drug resistance. ${ }^{12-16}$

As with ketoconazole, the results of MIC determinations with fluconazole must be interpreted with caution because the conditions under which the tests are performed have a marked effect on the results. ${ }^{17}$ For this reason, a high MIC on its own is not proof that treatment failure or relapse is due to drug resistance. More convincing evidence is the demonstration that the MIC for the most recent candida isolate is higher than that for isolates obtained earlier during treatment. If the latter have not been retained, it is important to test clinical isolates in parallel with sensitive and resistant reference strains of the same Candida sp. Most of the recent reports of apparent resistance to fluconazole of Candida spp. from AIDS patients have been based on isolated MIC determinations on strains obtained after treatment had failed; few have included comparisons with either earlier isolates from the same patient or with control strains.

In the case of $C$. albicans isolates from AIDS patients who failed to respond to treatment with increasing doses of fluconazole, MICs of ketoconazole and itraconazole have sometimes been found to be much lower than those of fluconazole. ${ }^{12-14}$ Less than ideal test conditions could account for these findings. Another explanation is selective resistance to fluconazole, but this seems less plausible because all azole antifungals have a similar mechanism of action.

In contrast to the long periods of treatment needed to induce azole resistance in $C$. albicans, short courses of fluconazole treatment have proved sufficient to induce resistance in C. glabrata (Torulopsis glabrata). Unlike $C$. albicans, this organism is haploid and this could explain the rapid development of clinical resistance noted in several recent reports. In one reported case, a $C$. glabrata isolate showed a 30 -fold rise in 
fluconazole MIC after 9 days of oral treatment ${ }^{18}$ further investigation indicated that this isolate was cross-resistant to itraconazole and ketoconazole. It is unclear whether resistance emerged as a result of the selection of a pre-existing sub-population of resistant organisms, or whether it was due to the development of drug-resistant mutants during treatment. The mechanism of resistance in C. glabrata appears to involve failure of the drug to penetrate the cell envelope (unpublished observations).

Another source of concern arising from the widespread use of fluconazole has been an apparent increase in the prevalence of colonisation and infection with intrinsically less sensitive yeasts, such as $C$. $k$ rusei. Fluconazole MICs for this species are, unlike $C$. albicans, often high before treatment. Recent case reports have described the failure of the drug to suppress $C$. krusei infection..$^{19-21}$ Other reports have suggested a higher incidence of both colonisation and infection with $C$. krusei among neutropenic patients who had received fluconazole than among similar patients who had not. ${ }^{22}$ Increased colonisation with $C$. glabrata was also noted in those receiving fluconazole, but there was no increase in the number of infections caused by this organism. In contrast, the number of serious infections caused by $C$. albicans and $C$.

\section{References}

1. Kerridge D. Mode of action of clinically important antifungal drugs. Adv Microb Physiol 1986; 27: 1-72.

2. Kerridge D, Nicholas RO. Drug resistance in the opportunistic pathogens Candida albicans and Candida glabrata. $J$ Antimicrob Chemother 1986; 18 Suppl B: 39-49.

3. Horsburgh CR, Kirkpatrick $\mathrm{CH}$. Long-term therapy of chronic mucocutaneous candidiasis with ketoconazole: experience with twenty-one patients. Am J Med 1983; 74 Suppl 1B: 23-29.

4. Warnock DW, Johnson EM, Richardson MD, Vickers CFH. Modified response to ketoconazole of Candida albicans from a treatment failure. Lancet $1983 ; 1$ : 642-643.

5. Smith KJ, Warnock DW, Kennedy CTC et al. Azole resistance in Candida albicans. J Med Vet Mycol 1986; 24: 133-144.

6. Johnson EM, Richardson MD, Warnock DW. In-vitro resistance to imidazole antifungals in Candida albicans. J Antimicrob Chemother 1984; 13 : 547-558.

7. Odds FC, Abbott AB. Relative inhibition factors-a novel approach to the assessment of antifungal antibiotics in vitro. J Antimicrob Chemother 1984; 13: 31-43.

8. Ryley JF, Wilson RG, Barrett-Bee KJ. Azole resistance in Candida albicans. J Med Vet Mycol 1984; 22 : 53-63.

9. Hitchcock CA, Barrett-Bee KJ, Russell NJ. Inhibition of $14 \alpha$ sterol demethylase activity in Candida albicans Darlington does not correlate with resistance to azole. $\mathrm{J} \mathrm{Med} \mathrm{Vet}$ Mycol 1987; 25: 329-333.

10. Tavitian A, Raufman J-P, Rosenthal LE, Weber J, Webber CA, Dincsoy HP. Ketoconazole-resistant Candida esophagitis in patients with acquired immunodeficiency syndrome. Gastroenterology 1986; 90: 443-445.

11. De Wit S, Weerts D, Goossens H, Clumeck N. Comparison of fluconazole and ketoconazole for oropharyngeal candidiasis in AIDS. Lancet 1989; 1 : 746-748. tropicalis was significantly lower in patients receiving prophylactic fluconazole. ${ }^{22}$

Treatment failure, attributable to the development of azole-resistant $C$. albicans strains, appears to be becoming more common, but still seems to be confined to patients receiving long-term treatment. In view of the increasing number of individuals requiring this form of management and the apparent potential of such treatment to select for resistance, it is becoming more important to document the magnitude of the problem and investigate the effects of changing prescribing practices. In the meantime, it must not be assumed that the development of fluconazole resistance among $C$. albicans strains during treatment is a characteristic of this particular drug not shared with other azole compounds. Until it is proved otherwise, it must be regarded as an inevitable consequence of prolonged drug use. The rapid development of drug resistance in patients with $C$. glabrata infection and the persistence of $C$. krusei infection observed in patients treated with fluconazole is also of concern and highlights the need for accurate identification of organisms before commencing treatment.

\section{W. WARNOCK}

Regional Mycology Laboratory, Department of Microbiology, Bristol Royal Infirmary, Bristol BS2 8HW.

12. Fox R, Neal KR, Leen CLS, Ellis ME, Mandal BK. Fluconazole resistant candida in AIDS. $J$ Infect $1991 ; 22$ : 201-204.

13. Kitchen VS, Savage M, Harris JRW. Candida albicans resistance in AIDS. $J$ Infect 1991 ; 22 : 204-205.

14. Smith D, Boag F, Midgley J, Gazzard B. Fluconazole resistant candida in AIDS. $J$ Infect $1991 ; 23$ : 345-346.

15. Willocks L, Leen CLS, Brettle RP, Urquhart D, Russell TB, Milne LJR. Fluconazole resistance in AIDS patients. $J$ Antimicrob Chemother 1991; 28: 937-939.

16. Dupont B. Antifungal therapy in AIDS patients. In : Bennett JE, Hay RJ, Peterson PK (eds) New strategies in fungal disease. Edinburgh, Churchill Livingstone. 1992; 290-300.

17. Odds FC. Laboratory tests for the activity of imidazole and triazole antifungal agents in vitro. Semin Dermatol 1985; 4 260-270.

18. Warnock DW, Burke J, Cope NJ, Johnson EM, Von Fraunhofer NA, Williams EW. Fluconazole resistance in Candida glabrata. Lancet 1988 ; 2 : 1310.

19. Case CP, MacGowan AP, Brown NM, Reeves DS, Whitehead P, Felmingham D. Prophylactic oral fluconazole and candida fungaemia. Lancet 1991; 337: 790.

20. McIlroy MA. Failure of fluconazole to suppress fungemia in a patient with fever, neutropenia, and typhlitis. I Infect Dis 1991 ; 163 : $420-421$.

21. Persons DA, Laughlin M, Tanner D, Perfect J, Gockerman JP Hathorn JW. Fluconazole and Candida krusei fungemia $N$ Engl J Med 1991; 325: 1315.

22. Wingard JR, Merz WG, Rinaldi MG, Johnson TR, Karp JE, Saral $\mathbf{R}$. Increase in Candida krusei infection among patients with bone marrow transplantation and neutropenia treated prophylactically with fluconazole. $N$ Engl $J$ Med 1991; 325: 1274-1277. 